\title{
Effect of Different Solvents and Ratio towards Microalgae Oil Production by Ultrasonic Assisted Soxhlet Extraction Techniques
}

\author{
Y. C. WONG ${ }^{1 *}$ and R. SHAHIRAH ${ }^{1}$ \\ ${ }^{1}$ Faculty of Bioengineering and Technology, Universiti Malaysia Kelantan, Jeli Campus, Locked Bag 100, \\ 17600 Jeli, Kelantan, Malaysia. \\ ${ }^{*}$ Corresponding author E-mail: yeeching@umk.edu.my \\ http://dx.doi.org/10.13005/ojc/350418
}

(Received: February 03, 2019; Accepted: August 03, 2019)

\begin{abstract}
Currently, the renewable feedstock is the most needed worldwide. Microalgae are promising raw materials for supplying biofuels due to the depletion of fossil fuels. The microalgae found were mostly consists of freshwater algae. The aim of this research is to study the effect of different solvents and ratio using Soxhlet extraction method. Many parameters were introduced in this study in order to enhance the lipid production of microalgae. Lipid content of algae oil production known as Fatty acid that found was being analyzed. Different solvents used and ratio was expected to have different highest in lipid content. Chloroform, ethanol, and hexane were chosen as the solvents used. The best solvent for lipid production was the combination of different solvents and ratio. Data showed that $8 \%$ is the highest total oil extraction yield obtained from combination of chloroform and ethanol with a ratio (1:2). The compound and lipid content in algae oil are analyzed through Gas Chromatography Mass Spectrometer (GC-MS) analysis. Fatty acids have many benefits and also have interest preparation for health products. Green microalgae strain was being identified and cultured as future potential for biodiesel production. Significant of this study is to unveil the benefit of algae oil as sustainable future resources.
\end{abstract}

Keywords: Microalgae oil, Ultrasonic, Extraction.

\section{INTRODUCTION}

The world energy crisis and increased greenhouse gas emissions have driven the search for alternative and environmentally friendly renewable energy sources ${ }^{1}$. The importance for identification of potential renewable source for sustainable energy production has gained momentum recently². The demand for sustainable energy nowadays has increase for a sustainable future. This study was conducted to deal with the renewable energy aspect focusing on the biomass from bioenergy crops to microalgae and increasing biofuel production ${ }^{3}$.

Microalgae biofuel is one of the major renewable energy sources for sustainable development and potentially to replace the fossilbased fuels ${ }^{4}$. Algae-based biofuels economically

This is an Open Access article licensed under a Creative Commons license: Attribution 4.0 International (CC- BY). Published by Oriental Scientific Publishing Company @ 2018 
viable and cost competitive, require minimal water use and mitigate atmospheric Carbondioxide. Microalgae can be found everywhere, from the oceans, lakes, rivers, soil, rocks, permanent snow and ice to desert ${ }^{4}$. These abilities make microalgae the most abundant living organisms on earth.

For this study, ultrasonic assisted soxhlet extractor was used to extract the algae oil from microalgae. Moreover, this research conducted to see the effect on different solvents and ratio in algae oil extraction towards microalgae. Chloroform, ethanol and hexane was chosen as the solvents used based on the polarity that suitable in extraction of lipid in algae. Polarity factors will influence the extraction of lipid content produced from the algae itself ${ }^{5}$. So, high production of algae oil will be increasing for further uses. GC-MS. (Gas Chromatography Mass Spectrometry) is used to determine and analyzed the components in the algae oil which obtained from the extraction process.

\section{MATERIALS AND METHODS}

\section{Sampling}

Freshwater was collected at fish pond UMK Jeli campus. GPS Coordinates (05 $44-48.2^{\prime \prime}$, 10152.-05.6") Latitude, Longitude. A microalgal strain was taken because it is most attractive microalgae species for oil extraction as its easy found and easily to be managed ${ }^{6}$.

The strain became the most favored by researchers since it has a high lipids content and easily growth in the laboratory? ${ }^{7}$. The sample was collected in a $1.5 \mathrm{~L}$ mineral bottle. Samples were sent to the laboratory for further studies.

\section{Identification of microalgae in fish pond}

Fresh water sample collected was being analyzed using light microscope 10X and 40X powers for general identification of algae existed in the water sample ${ }^{8}$. The morphology of microalgae species was known as based on its structure and shape.

\section{Culture of microalgae}

Microalgae strain was cultivated in batches culture in a $1 \mathrm{~L}, 2 \mathrm{~L}$ and $5 \mathrm{~L}$ of media bottles. Fluorescent lamps were used to control different light intensity at 85,2800 and 6800 . Light intensity for normal light (NL) is 6800 lux, for reduce light (RL) is 28000 lux and for no light (NOL) (media covered with aluminum foil) is 85 lux. The temperature of $25^{\circ}$ $\mathrm{C}$ and $\mathrm{pH}$ around 6.5 of culture were maintained and controlled same like its previous environment. Aeration that supply with $\mathrm{CO}_{2}$ was given to the cultures in order to maintain $\mathrm{pH}$ below 8 .

\section{Harvesting of microalgae}

Centrifuge machine was used at 5000 rpm (rotate per minutes) for 15-20 min with room temperature $^{9} .1 \mathrm{~L}, 2 \mathrm{~L}$, and $5 \mathrm{~L}$ cultures of microalgae from different light sources were harvested. Two-layer was formed (supernatant and pellet) after was centrifuge. Supernatant was thrown away and the pellet was washed three times with distilled water. Microalgae were cultivated with new batches after centrifugation processes.

\section{Drying}

The pellet of algae was poured into a plastic petri dish and dried overnight using an oven at $60^{\circ} \mathrm{C}$ for 12-18 hours. The dried pellet was milled using mortar. Ziploc bags were used to store the dried pellet and it was kept in a desiccator. Desiccator was used to preserve the dried pellet.

\section{Extraction of oil}

$10 \mathrm{~g}$ of powdered algae was used for the Soxhlation method. Each of the Soxhlet extractors was filled with different solvents and ratio. The solvent was heated for $6-8 \mathrm{~h}$ at $50-70^{\circ} \mathrm{C}^{8}$. In order to remove the solvent from the bio-crude oil, the extracts were concentrated through evaporation using rotary evaporator. Crude oil was stored at fume chamber in a dry airtight container.

\section{Separation of algae oil from crude}

For pretreatment, the sonification process was undergoing. Hexane was added as a solvent to separate the oil from the particle. After that, centrifuge was done into $2 \mathrm{~mL}$ centrifuge tubes each of the samples then put into microliter centrifuge at $10000 \mathrm{rpm}$ within $10-15 \mathrm{~min}^{10}$. After centrifuge, two layers were formed and it was washed away several times. The density of solvents was used to determine the layer of oil.

\section{Analysis using GC-MS}

Algae oils were analyzed by using Gas Chromatography Mass Spectrometer (GC-MS) to 
obtain the lipid contents. The samples were put into vial bottle that is special bottle for using GC-MS. The samples were injected into injector by GC-MS. The temperature of injection port was maintained at $80^{\circ} \mathrm{C}$ for $4 \mathrm{~min}$ and it was increased to $10^{\circ} \mathrm{C}$ per $\mathrm{min}^{11}$. The overall temperature value of the column was $240^{\circ} \mathrm{C}$ and the runtime for each sample were about 45 minutes.

\section{Solvent system calculation}

There are 3 different types of solvents that were used consists of polar and non-polar solvents. Non-polar solvents such as chloroform and hexane and polar solvents such as ethanol were used. Chloroform was used $300 \mathrm{~mL}$ as the solvents in Soxhlet Extraction for the first extraction. Next, the combination of different solvents with different polarity and ratio were used. Combination of chloroform and ethanol with a ratio (1:1) means that $150 \mathrm{~mL}$ of chloroform and $150 \mathrm{~mL}$ of ethanol were used as the solvents in Soxhlet Extraction ${ }^{8}$. Same values of solvent were applied to the combination of chloroform and ethanol with a ratio (1:1). For the combination of chloroform and ethanol with a ratio (1:2) means that $100 \mathrm{~mL}$ of chloroform and $200 \mathrm{~mL}$ of ethanol were used as the solvents in Soxhlet Extraction. Same values of solvent were applied to the combination of chloroform and hexane with a ratio (1:2).

\section{RESULTS AND DISCUSSION}

\section{Effects of light on Algae growth}

Microalgae from fish pond UMK Jeli were extracted using different solvents and ratio that is chloroform, ethanol, and hexane. This study was done in order to analyze the total lipid contents in the algae oil. The total lipid contents in this study were measured using GC-MS method.

Figure 1 shows the production of algae dries biomass for normal light (NL), reduce light (RL), no light (NOL). The results show significantly different in three types of cultures. Light intensity also plays a major role in the production growth of algae. Some light intensity can enhance or can disrupt the performance growth of algae.

Based on the result, the highest production of algae was reduced light (2800 lux) at $95.78 \mathrm{~g} / \mathrm{L}$. The lowest production of algae was no light (85 lux) at $27.43 \mathrm{~g} / \mathrm{L}$ followed by normal light (6800 lux) at $62.56 \mathrm{~g} / \mathrm{L}$. The effect of light intensity has been studied on the cultivation of microalgae. The growth of microalgae was dependent on the light intensity provided. The cultivation of microalgae has been done for about one week before harvested. Dried microalgae were collected for extraction of algae oil. As the previous study by Rohit and Mohan ${ }^{12}$ shows that such a long lighting period will increase the C. vulgaris growth which is $(2.025 \mathrm{~g} / \mathrm{L})$ of the maximum biomass is obtained in 5 Klux.

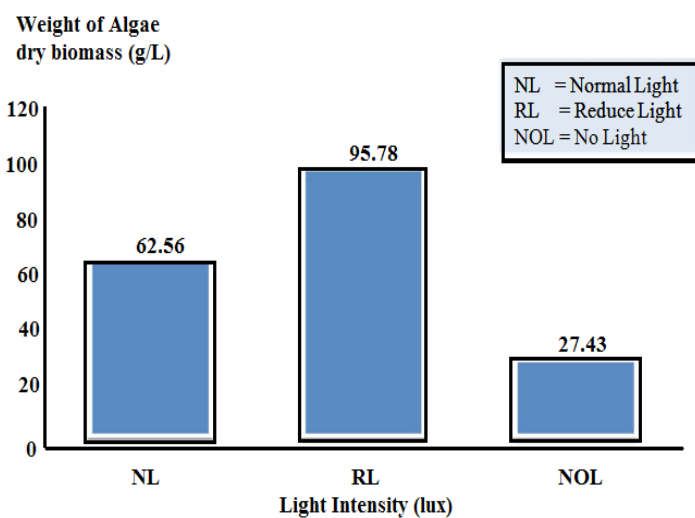

Fig. 1. The production of algae dry biomass

According to Rohit and Mohan ${ }^{12}$ the production of chlorophyll content in the microalgae was influenced by light and it causes effects in the absence of energy sources such as light and organic carbon. Research in the previous study by Daliry et al., ${ }^{13}$ some findings suggest a non-continuous light intensity due to growth rates are remain high and production costs are reduced. It happens because cell division usually occurs under dark conditions for single cell photosynthetic culture.

In some cases, dark and light phases were required for cell division, but for $C$.vulgaris, more cell division occurs after stopping the lighting phase. Normally, in laboratory scale microalgae production, its use of fluorescent light which has light or dark cycle but in this study, artificial light was provided for 24 hours. Lux meter was used to measure the different light intensity. According to Rai et al., ${ }^{14}$ the growth of Chlorella sp. was influenced by light intensity. The maximum growth rate of microalgae can increase when there is enough light intensity such as at 2700 lux. For this experiment, it can be concluded that the higher the light intensity for microalgae cultivation, the lower the production of dried biomass. 


\section{Extraction of microalgae oil Ultrasonic techniques}

In this study, the time taken for the sonication was set at 20 min with 5 min time lapse. Time lapse is important in this process because the solvents used have high kinetic energy when contacting with pressure. To prevent the high temperature increase, so time lapse was applied in this experiment. The extraction yield increase as the extraction time increased ${ }^{15}$. Longer time gave the cell walls more time to disrupt hence the releasing of cell contents more efficiently. But to long extraction time will make the extraction solution became saturated and the increasing in yield of oil were possible after saturation. As the previous study by Zhan et al., ${ }^{15}$ shows that 20 min was selected as the optimum extraction time for subsequent experiments.

\section{Soxhlet extraction techniques}

For the extraction process, all the dried sample were extracted using different solvents and ratio. All samples was carried out using the Soxhlet extraction method as described by Olusegun \& Philip $^{16}$ and the sample was extracted for $8 \mathrm{~h}$ in this experiment. Based on researcher done by Nurfarahanim et al., 87 to $8 \mathrm{~h}$ is the best time to do extraction because between this time intervals, the oil was found at the maximum yield and at extraction time above $8 \mathrm{~h}$ the oil did not show any more significant change in the extraction. Different solvent and ratio were extracted using chloroform, ethanol, and hexane. Lipid extraction in algae oil can enhance by increasing the polarity of the solvent by mixing the polar and non-polar organic solvents. This happens because due to releasing of lipid from the microalgae protein-lipid complexes by the ability of the polar solvents.

\section{Fatty acid profile of triglycerides extracted from microalgae}

The fatty acid (FA) fractions were analyzed by GC (Agilent Technologie model 7890 B) with flame ionization detector and capillary column MS (Agilent Technologies 5977 A MSD) 25 m length $\times 0.32 \mathrm{~mm}$ internal diameter $\times 0.3 \mu \mathrm{m}$ thickness as refers to the previous study by Prommuak et al., (2012). Only 5 samples from normal light were chosen because all the sample from normal light shows greatest algae oil production. There are C, C:E (1:1), C:E (1:2), C:H (1:1), and C:H (1:2). According to Prommuak et al., 18 fatty acid profile of microalgae was obtained from
GC-MS analysis of its bio-oil composition of algae oil. The results of fatty acid profile extracted from these samples microalgae were agreed with Nayak et al., ${ }^{18}$ that reported the most abundant FAME from microalgae was methyl linoleate and methyl palmitate followed by methyl oleate.

For fatty acid chains, saturated fatty acid (SFA) refers to the simplest straight-chain. Fatty acids are known as unsaturated normally called monounsaturated (MUFA) if double bonds present. Polyunsaturated (PUFA) is known as one double bond. Fig. 2 shows the most representative for Saturated Fatty Acid (SFA) that known as fatty acid of carbon single bond. It consists of Hexadecanoic Acid Methyl Ester (HAME) and Hexadecanoic Acid Ethyl Ester (HAEE) which commonly known as palmitic acid, butanoic acid, myristic acid, and stearic acid.

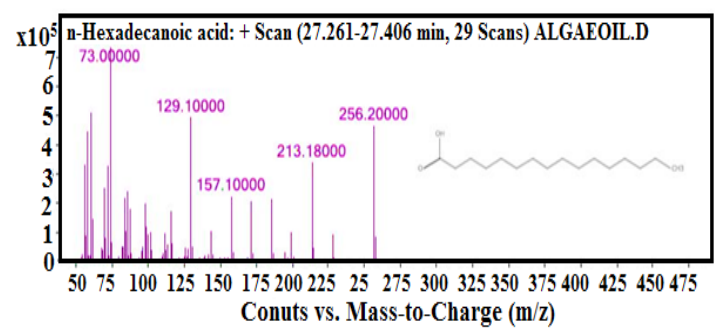

Fig. 2a. Saturated Fatty Acid (SFA) found in Algae oil

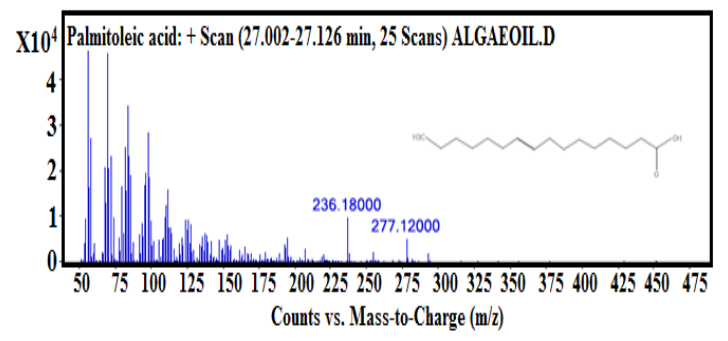

Fig. 2 b. The production of algae dry biomass

Table 1 shows the fatty acid profile of the microalgae studied and some sources of common lipids. This sample was extracted using chloroform as the solvent. For this sample, the upper part was being referred. Sample from chloroform was not clearly obtained it oily layer because only $2 \%$ of oil was obtained from total algae biomass sample. Palmitic acid normally known as a source of animal fat. The acid has higher content of C16:0 (21.79\%) followed by C18:0 (oleic acid : $3.16 \%$ ), for C16:0 (n-hexadecanoic acid : 1.69) and C18:0 (6-octadecanoic acid : $1.08 \%$ ). There is another fatty acid that was found in this sample because have effected by the solvent used in the process. 
Table 1: Fatty acid profile of Triglycerides extracted from Chloroform of Microalgae

\begin{tabular}{lcccc}
\hline Common name & Fatty acid & Peak & RT $(\min )$ & Area (\%) \\
\hline Palmitic acid & C16:0 & 5 & 27.385 & 21.79 \\
Oleic acid & C18:0 & 12 & 31.204 & 3.16 \\
n-hexadecanoic acid & C16:0 & 6 & 28.231 & 1.69 \\
6-octadecanoic acid & C18:0 & 11 & 30.826 & 1.08 \\
\hline
\end{tabular}

By referring to Table 2, this sample was extracted from chloroform and ethanol by a ratio of 1:1. In this study, C16:0 (Palmitic acid : 19.75\%) have its higher content of lipid, followed by $\mathrm{C} 18: 0$ (Oleic acid : $6.78 \%$ ), C16:0 (n-hexadecanoic acid : 1.45) and C18:0 (6-octadecanoic acid : 1.14\%). This sample got second highest production of oil because the combination of polar and non-polar solvents can extract highest yield of oil.

Table 2: Fatty acid profile of triglycerides extracted from C:E (1:1) of Algae

\begin{tabular}{lcccc}
\hline Common name & Fatty acid & Peak & RT (min) & Area (\%) \\
\hline Palmitic acid & C16:0 & 3 & 23.587 & 19.75 \\
Oleic acid & C18:0 & 6 & 34.698 & 6.78 \\
6-octadecanoic acid & C12:0 & 14 & 30.645 & 1.14 \\
n-hexadecanoic acid & C16:0 & 5 & 28.563 & 1.45 \\
\hline
\end{tabular}

Table 3 shows a significantly different trend of lipid content in this sample where the sample refers to the lower part of algae oil. This sample extracted from chloroform and ethanol with a ratio (1:2). In this study, C18:0 (6-octadecanoic acid: $99.15 \%$ ) that usually found in olive oil was the highest lipid content followed by C16:0 (n-hexadecanoic acid: $20.77 \%$ ), C16:0 (Hexadecanoic acid: 1.96\%) and C18:0 (oleic acid: 1.26). On the other hand, n-dodecanoic acid that also known as lauric acid $\mathrm{C} 12: 0$ was found in this sample. Lauric acid is commonly found in coconut oil and microalgae cells also have that kind of lipid.

Table 3: Fatty acid profile of triglycerides extracted from C:E (1:2) of Algae

\begin{tabular}{lcccc}
\hline Common name & Fatty acid & Peak & RT (min) & Area (\%) \\
\hline 3-octadecenoic acid & C18:0 & 8 & 28.901 & 1.88 \\
6-octadecanoic acid & C18:0 & 11 & 29.679 & 99.15 \\
Hexadecanoic acid & C16:0 & 7 & 27.169 & 1.96 \\
n-dodecanoic acid & C12:0 & 6 & 27.459 & 20.77 \\
Oleic acid & C18:0 & 5 & 27.212 & 1.26 \\
\hline
\end{tabular}

In this study, the sample was extracted from chloroform and hexane ratio of (1:1). From Table 4 , it can be shown that the C16:0 (6-octadecanoic acid: $17.63 \%$ ) that usually found in olive oil has the highest yield followed by C16:0 (3-octadecenoic acid, $1.63 \%$ ), oxiraneundecanoic acid $1.59 \%$, oleic acid $1.14 \%$ and 2,5 octadecadiynoic acid 1.14. Besides, at peak 9 with retention time $27.448 \mathrm{~min}$ in the GC-MS, Alpha-Linoleic acid (ALA) was be found. Alpha-Linoleic acid is an unsaturated Essential Fatty Acid (EFA) that also known as omega-3 fatty acid.

Table 4: Fatty acid profile of triglycerides extracted from C:H (1:1) of Algae

\begin{tabular}{lcccc}
\hline Common name & Fatty acid & Peak & RT (min) & Area (\%) \\
\hline Oleic acid & C18:0 & 10 & 28.849 & 1.14 \\
3-octadecenoic acid & C16:0 & 11 & 28.906 & 1.63 \\
6-octadecanoic acid & C16:0 & 17 & 29.975 & 17.63 \\
Alpha-Linoleic acid (ALA) & C18:3 & 9 & 27.448 & 1.14 \\
Oxiraneundecanoic acid & C16:0 & 7 & 26.747 & 1.59 \\
\hline
\end{tabular}

By referring to Table 5, it shows n-hexadecanoic acid have the highest lipid content for chloroform and hexane solvents with a ratio (1:2). It was found at peak 7 with a retention time 27.437 minutes. Followed by C18:0 (Oleic acid: $1.64 \%$ ) at peak 18 with retention time 32.076 min. Oleic acid was commonly found in olive oil component and algae cells also contained it. This sample also contained C16:0 (hexadecanoic acid : 1.46\%), 6-octadecanoic acid $1.41 \%$ and palmitic acid $1.11 \%$.

Table 5: Fatty acid profile of triglycerides extracted from C:H (1:2) of Algae

\begin{tabular}{lcccc}
\hline Common name & Fatty acid & Peak & RT (min) & Area (\%) \\
\hline 6-octadecanoic acid & C16:0 & 11 & 30.452 & 1.41 \\
Palmitic acid & C16:0 & 9 & 28.87 & 1.11 \\
Hexadecanoic acid & C16:0 & 8 & 27.437 & 1.46 \\
n-hexadecanoic acid & C16:0 & 7 & 27.437 & 31.07 \\
Oleic acid & C18:0 & 18 & 32.076 & 1.64 \\
\hline
\end{tabular}

The presence of fatty acid in all sample and different in fatty acid profiling due to the method of extraction used and also the effect of different light intensity applied to the sample. Some of the fatty acid that presents in all sample of algae oil suitable for biodiesel study ${ }^{19}$.

\section{Effect of different solvent and ratio of algae oil \\ The solvents used for the extraction} microalgae biomass showed the different yields on production of oil percentages. Based on Fig. 3, 8\% is the highest total oil extraction yield obtained from combination of chloroform and ethanol with a ratio (1:2). Approximately, 6\% total oil extraction yield obtained using chloroform and hexane with a ratio (1:1).Whereas $5 \%$ oil extraction yield was obtained using chloroform and hexane with a ratio (1:2). The oil extraction yield for chloroform and hexane with a ratio (1:1) was found to be $4 \%$ and $2 \%$ respectively. 


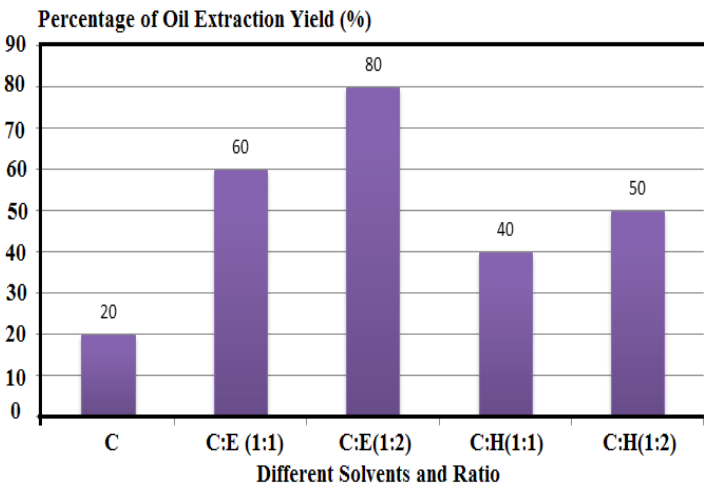

Fig. 3. Oil extraction yield with different solvent systems

It is an important factor to select the solvent system for oil extraction in order to produce the highest yield of oil. Solvent system should have a higher solubility with oil to degrade the cell walls of the microalgae and also to dissolve the oil to enhance the oil yield. The efficiency of oil extraction yield depends on the polarity of the compound and solvent viscosity ${ }^{20}$. Different solvents and ratio effect different yield production of algae oil.

The selected solvent came from polar and non-polar solvents. These solvents have high dielectric and constant and high polarity. Non-polar solvents contain bonds between atoms with similar electronegativity ${ }^{20}$ such as carbon and hydrogen. Similar electronegativity's mean that small difference between atoms causing less polar bond, thus easier to extract the oil. Alkaline like hexane and heptane are examples for this non-polar solvent ${ }^{21}$.

Based on researcher done by Conkerton et al.,22 heptane is the best alternative solvent to replace hexane since the physical properties of heptane is quite similar to hexane. However, when the hexane is combined with other solvents to extract the oil, the yield of oil extraction becomes low. This observation is the same as the study of Ryckebosch et al., ${ }^{23}$ they stated that combination non-polar and polar solvent with proper ration can extract a high yield of oil. The experimental result obtained is contrasted with Shen et al., ${ }^{24}$ that stated oil recovery was higher in the single solvent. A possible cause for the lower oil recovery efficiency in mixture solvent may be that small quantities of oils take over by the polar solvent that cannot be extracted by hexane. Combination of solvents systems between polar and non-polar can be used as long as it is implied at appropriate proportions of polar and non-polar solvents in order to achieve high oil extraction yields.

\section{CONCLUSION}

This study was carried out to study the effects of different solvent and ratio on the production of oil from microalgae. Different light intensity also was carried out on the growth of microalgae. The difficulty of using algae in this study is algae took times to grow, sometimes even after 7 days, the algae did not grow hence more time needed. After culturing, the algae were harvested using a centrifuge. After harvested, only $10 \%$ of pure algae managed to obtain from $5 \mathrm{~L}$ of media. $0.03 \mathrm{~g}$ of dry algae was collected from $5 \mathrm{~L}$ of algae. A small quantity of dry algae was obtained after harvested because the dry algae were difficult to harvest and the rest of it contained water. For the fatty acid profile of triglycerides extracted from microalgae, there is no difference on composition with difference solvents and ratio on fatty acid production.

The oil extracted using Soxhlet method contains many new discoveries of compounds that can be used in further study. Overall, it can be concluded that algae oil is very precious oil that can contribute to the production of biofuel. Algae are recommended to be used because algae are abundant in the environment and it is easy to get. Apart from that, algae oil also useful in pharmaceutical, health and beauty industries. For example, algae oil contains oleic acid that useful as a high antioxidant and anti-inflammatory compound.

\section{ACKNOWLEDGEMENT}

The authors express their sincere appreciation to the Faculty of Bioengineering and Technology, Universiti Malaysia Kelantan, Jeli Campus for the support and good facilities which made this study possible.

\section{Conflict of interest}

Authors declare no conflict of interest.

\section{REFERENCES}

1. Medipally, R. S; Fatimah, M.Y; Banerjee, S. M; Shariff. Microalgae as Sustainable Renewable Energy Feedstock for Biofuel Production. Plant
Biotechnology for Sustainable Production of Energy. J. BioMed. Research. International., 2015, 15, 9-13. 
2. Lum, K. K; Kim, J; Lei, X. G. Dual potential of microalgae as a sustainable biofuel feedstock and animal feed. J. Animal. Science. Biotechnology., 2013, 4, 1.

3. Ndimba, B, K; Ndimba, J. R; Johsan, S, T; Baba, M, Sirisattha, S; Shiraiwa, Y; Agrawal, K, G; Rakwal, R. Biofuels as a Sustainable Energy Source: An Update of The Application of Proteomics in Bioenergy Crops and Algae. J. Proteomics., 2015, 93, 234-244.

4. Katiyar, R. G.B. R; Biswas, S; Pruthi, V; Kumar, $\mathrm{N}$, Kumar, P. Microalgae: An emerging source of yenergy based bio-products and a solution for environmental issues. Renewable and Sustainable Energy Reviews., 2016.

5. Sharma, K. K; Schuhmann, H; Schenk, P. M. High Lipid Induction in Microalgae for Biodiesel Production. J. Energies., 2012, 5, 1532-1553.

6. Al-Iwayzy, S. H; Yusaf, T. Al-Juboori, R. A. Biofuels from the fresh water microalgae Chlorella vulgaris (FWM-CV) for diesel engines. J. Energies., 2014, 7, 1829-1851.

7. Ahmad, A. L; Yasin, N. H. M; Derek, C. J. C; Lim, J. K (2014). Comparison of harvesting methods for microalgae Chlorella sp. And its potential use as a biodiesel feedstock. J. Environmental. Technology., 2014, 35, 2244-2253.

8. Nurfarahanim, A; Nur, A; Hidayah, M. Algae oil extraction from freshwater microalgae Chlorella vulgaris. J. Malaysian. Analytical. Sciences. Society., 2017, 3, 735-744.

9. Saad, B; Brandon, B; Chew, C; Georgios, K; Aanchal; Manu. Hand-powered ultralow-cost paper centrifuge. J. Nature., 2017, 18-23.

10. Hiroaki, I; Yuji, A; Tsunao, K; Masaharu, S. Sonoporation-mediated transduction of siRNA ameliorated experimental arthritis using 3 MHz pulsed ultrasound. J. Ultrasonics., 2014, 54, 874-881.

11. Hussain, S.Z; Maqbool, K. GC-MS: Principle, Technique and its application in Food. $J$. Current. Science., 2014, 13, 116-126.

12. Rohit, M. V; Mohan, S. V. Tropho-metabolic transition during Chlorella sp. cultivation on synthesis of biodiesel. J. Renewable. Energy., 2016, 1-8.

13. Daliry, S; Hallajisani, A; Roshandeh, J. M; Nouri, H; Golzary. Investigation of optimal condition for Chlorella vulgaris microalgae growth. J. Environ. Sci. Manage., 2017, 3,
217-230.

14. Rai, M. P; Gautom, T; Sharma, N. Effect of Salinity, pH, Light Intensity on Growth and Lipid Production of Microlagae for Bioenergy Aplication. J. Biological. Sciences., 2015, 15, 260-267.

15. Zhan.J.L;Feng.J.Y;Yang.L;Yuan. G.Z.Ultrasonic Extraction of Oil from Caesalpinia spinosa (Tara) Seeds. J. Chemistry., 2016, 2, 6.

16. Olusegun, A; Phillip, A. Extraction of Oil from Microalgae And Aquatic Plants Harvested From Fish Rearing Structures. J. Science. Technology., 2014, 4, 45-47.

17. Prommuak, C; Pavasant, P; Quitain, A. T; Goto, M. Microalgal lipid Extraction and Evaluation of Single-Step Biodiesel Production., 2012, 26-27.

18. Nayak, M; Suh, W. I; Lee, B; Keun, Y. Enhanced carbon utilization effiency and FAME production of Chlorella sp. Energy Conversation and Management., 2018, 45-52.

19. Maulidiyah; Nurdin, M.; Fatma , F.; Natsir , M.; Wibowo, D. Characterization of methyl ester compound of biodiesel from industrial liquid waste of crude palm oil processing. J. Analytical Chemistry Research., 2017, 12. 1-9.

20. Rezaie, M; Farhoosh, R; Iranshahi, M; Sharif, A; Golmohamadzadeh, S. Ultrasonic- assisted extraction of antioxidative compounds from Bene (Pistacia atlantica subsp. Mutica) hull using various solvents of different physicochemical properties. Food Chemistry., 2015, 173, 577 - 583.

21. Reichardt, C; and Welton, T. Solvents and solvent effects in organic chemistry., 2011, 50, 234-235.

22. Conkerton, E. J; Wan, P. J; Richard, O. A (1995). Hexane and heptane as extraction solvents for cottonseed: A laboratory-scale study. J. American. Oil. Chemical. Society., 1995, 72, $963-965$.

23. Ryckebosch, E; Myuylaert, K; Foubert, I. Optimisation of an analytical procedure for extraction of oils from microalgae. J. American. Oil. Chemical. Society., 2012, 89, 189 - 198.

24. Shen, Y; Pei, Z. J; Yuan, W. Q; Mao, E. R. (2009). Effect of nitrogen and extraction method on algae oil yield. J. Agriculture. Biology. Engineering., 2009, 2, 51- 57. 